\title{
Supersymmetric Higgs boson pair production at hadron colliders
}

\author{
A. Belyaev,${ }^{1,2}$ M. Drees, ${ }^{1}$ O. J. P. Éboli, ${ }^{1}$ J. K. Mizukoshi, ${ }^{3}$ and S. F. Novaes ${ }^{1}$ \\ ${ }^{1}$ Instituto de Física Teórica, Universidade Estadual Paulista, Rua Pamplona 145, 01405-900, São Paulo, Brazil \\ ${ }^{2}$ Skobeltsin Institute for Nuclear Physics, Moscow State University, 119899 Moscow, Russia \\ ${ }^{3}$ Stanford Linear Accelerator Center, University of Stanford, Stanford, California 94309
}

(Received 7 May 1999; published 9 September 1999)

\begin{abstract}
We study the pair production of neutral Higgs bosons through gluon fusion at hadron colliders in the framework of the minimal supersymmetric standard model. We present analytical expressions for the relevant amplitudes, including both quark and squark loop contributions, and allowing for mixing between the superpartners of left- and right-handed quarks. Squark loop contributions can increase the cross section for the production of two $C P$-even Higgs bosons by more than two orders of magnitude, if the relevant trilinear soft breaking parameter is large and the mass of the lighter squark eigenstate is not too far above its current lower bound. In the region of large $\tan \beta$, neutral Higgs boson pair production might even be observable in the $4 b$ final state during the next run of the Fermilab Tevatron collider. [S0556-2821(99)05819-1]
\end{abstract}

PACS number(s): 14.80.Cp, 14.80.Ly

\section{INTRODUCTION}

The electroweak symmetry breaking sector is the last ingredient of the standard model (SM) that remains to be explored experimentally. If the masses of the $W$ and $Z$ bosons, as well as of the charged leptons and quarks, are due to the vacuum expectation value of an elementary scalar Higgs field, naturalness arguments indicate [1] that nature should become supersymmetric at an energy scale not far above the weak scale; otherwise quantum corrections would destabilize the hierarchy between the electroweak scale and the much larger Planck, ground unified theory (GUT) or string scale. Supersymmetry not only demands the introduction of superpartners for all known SM particles, but it also requires the existence of at least two Higgs doublets (and their superpartners). In its minimal version, the so called minimal supersymmetric standard model (MSSM) [2], there are three physical neutral Higgs bosons after electroweak symmetry breaking. In this work we assume that $C P$ is conserved in the Higgs bosons and squark sectors of the theory, and consequently the neutral Higgs bosons can be classified as two $C P$-even states $h, H$ (with $m_{h}<m_{H}$ ) and one $C P$-odd state A.

The production and detection of neutral Higgs bosons at $e^{+} e^{-}$colliders is quite straightforward [3]. It can be shown [4] that at least one MSSM Higgs boson would have to be found at an $e^{+} e^{-}$collider operating at a center-of-mass energy $\sqrt{s} \gtrsim 250 \mathrm{GeV}$. However, there are no immediate prospects for the construction of such a collider, and the energy of the CERN $e^{+} e^{-}$collider LEP may not suffice to find even one MSSM Higgs boson. On the other hand, the Fermilab Tevatron will soon begin its next collider run, at a slightly increased energy $(\sqrt{s}=2 \mathrm{TeV})$ and greatly increased luminosity (anticipated $\int \mathcal{L} d t=2 \mathrm{fb}^{-1}$ per experiment); future runs with yet higher luminosity are being contemplated. In a few years experiments at the CERN Large Hadron Collider (LHC) will also start taking data at $\sqrt{s}=14 \mathrm{TeV}$ and luminosity between 10 and $100 \mathrm{fb}^{-1} / \mathrm{yr}$. It is therefore important to explore all channels that might yield information about the
Higgs boson sector at hadron colliders.

The largest contribution to the inclusive neutral Higgs boson production cross section at such colliders comes from gluon fusion, which can produce a single Higgs boson through quark $[5,6]$ or squark [6] loops. The next-to-leading order (NLO) QCD corrections (involving gluons and light quarks) to these processes have already been computed $[7,8]$. On the other hand, NLO supersymmetric (SUSY) QCD corrections (involving gluinos) have not yet been calculated, however, they are expected to be smaller. One drawback of this production channel is that it can give a visible signal only if the Higgs boson decays into a mode with a small branching ratio. For example, in case of the light $C P$-even scalar $h$, the only promising decay mode in this production channel is $h \rightarrow \gamma \gamma$, which has a branching ratio of the order of $10^{-3}$ [9]. Moreover, if squark loop contributions are large, they tend to be destructive [10], at least for $g g \rightarrow h \rightarrow \gamma \gamma$. As a result, $g g \rightarrow \phi$ production in the MSSM $(\phi=h, H, A)$ cannot give a viable signal at the Tevatron, and is often difficult to detect even at the LHC.

One alternative is to search for the production of a single Higgs boson produced in association with some other particle(s) [11]. However, the main (hadronic) decay channels of MSSM Higgs bosons seem to be detectable in this way only in $W h(Z h)$ production at the Tevatron [12] if more than $10 \mathrm{fb}^{-1}$ of data can be accumulated, as well as in some cases for $h$ bosons produced in the cascade decays of heavy sparticles at the LHC [13]. The detectability of hadronic Higgs boson decays in $t \bar{t} \phi$ production [14] still awaits confirmation by an experimental study; no background estimates for the recently suggested [15] $\widetilde{t} \widetilde{t} \phi$ channels exists as yet. Finally, for very large values of $\tan \beta$ associate Higgs boson $b \bar{b}$ production might also be observable at the Tevatron in the $4 b[16]$ and/or the $b \bar{b} \tau^{+} \tau^{-}[17]$ mode.

In this paper we instead study the production of two neutral Higgs bosons. It is hoped that the $4 b$ final state, with invariant mass peaks in both $b \bar{b}$ pairs, will give a detectable signal at the Tevatron and/or the LHC at least in some regions of parameter space. Moreover, the Higgs boson pair 
production can in principle be used to probe the Higgs boson self-couplings in order to reconstruct its potential [18]. This process has first been discussed in Refs. [19] for the SM, and in [20] the quark loop contribution in the MSSM has been studied. NLO QCD corrections to heavy quark loops have also recently been calculated [21]; they are of similar relative size as the corresponding NLO corrections to single Higgs production. Here we extend these analyses by including the contribution from squark loops, allowing for general mixing of the superpartners of left- and right-handed quarks. We find that, unlike for single Higgs production [10], squark loop contributions can increase the total cross section by more than two orders of magnitude. In some regions of parameter space beyond the reach of LEP, the $4 b$ final state might even give a detectable MSSM Higgs signal at the next run of the Tevatron.

The primary purpose of this paper is to present all analytical expressions required for a calculation of the squark loop contribution to the production of two neutral Higgs bosons, and to illustrate their potential importance with a few numerical examples.

The remainder of this paper is organized as follows. In Sec. II we give analytical expressions for all one-loop amplitudes of the form $g g \rightarrow \phi_{i} \phi_{j}$, where $\phi_{i, j}$ are neutral Higgs bosons with definite $C P$ quantum numbers. These expressions are completely general; a list of the relevant couplings as predicted by the MSSM is given in the Appendix. In Sec. III some numerical results are shown. We focus on scenarios with either a small or a large value of the parameter $\tan \beta$, which governs the size of Yukawa couplings in the MSSM. We find potentially very large squark loop contributions in both cases, but the experimental discovery of Higgs boson pairs at the Tevatron seems to be somewhat more promising at large $\tan \beta$. Finally, Sec. IV is devoted to a brief summary and some conclusions.

\section{CROSS SECTIONS AND MATRIX ELEMENTS}

We write the subprocess (parton-parton) differential cross section as

$$
\frac{d \hat{\sigma}}{d \hat{t}}=\frac{|M|^{2}}{16 \pi \hat{s}^{2}}
$$

where the hatted Mandelstam variables refer to the partonparton system. The total cross section can be obtained from Eq. (1) by integrating over $\hat{t}$ and convoluting with the gluon densities in the two colliding hadrons, as usual. In general, the invariant amplitude can be written as

$$
\begin{aligned}
|M|^{2}= & 2\left(\frac{1}{4}\right)\left(\frac{1}{64}\right) S_{F}\left[\left|\sum_{n} M_{++}^{(n)}\left(\phi_{i} \phi_{j}\right)\right|^{2}\right. \\
& +\left|\sum_{n} M_{--}^{(n)}\left(\phi_{i} \phi_{j}\right)\right|^{2}+\left|\sum_{n} M_{+-}^{(n)}\left(\phi_{i} \phi_{j}\right)\right|^{2} \\
& \left.+\left|\sum_{n} M_{-+}^{(n)}\left(\phi_{i} \phi_{j}\right)\right|^{2}\right] .
\end{aligned}
$$
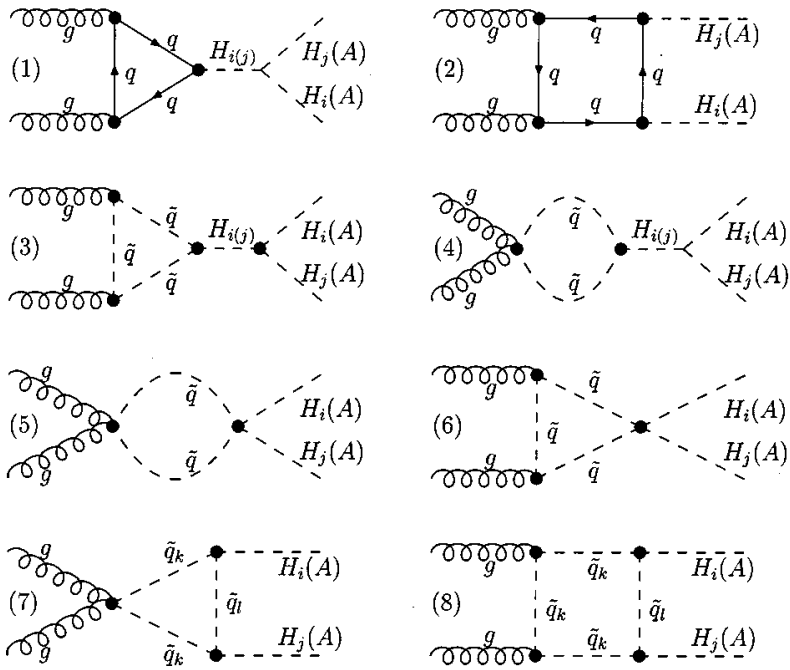

FIG. 1. Feynman diagrams for $h h, H H, h H$, and $A A$ Higgs boson pair production. $H_{i(j)}=h, H$ for $i(j)=1,2$, respectively, $\tilde{q}_{k(l)}=\tilde{q}_{1}, \tilde{q}_{2}$ for $k(l)=1,2$. The crossed diagrams are not shown.

Here $M_{\lambda_{1}, \lambda_{2}}^{(n)}\left(\phi_{i} \phi_{j}\right)$ is the helicity amplitude for the production of the Higgs boson pair $\left(\phi_{i} \phi_{j}\right)$ for the initial gluon helicities $\lambda_{1(2)}= \pm$. The sum runs over all Feynman diagrams (n) that contribute to a specific process. The factors refer to the average over the initial gluon helicities $\left(\frac{1}{4}\right)$, the color factor $\left[\operatorname{Tr}\left(T^{a} T^{b}\right)\right]^{2}=\frac{1}{4} \delta_{a a}=2$, and the average over the gluon colors $\left(\frac{1}{64}\right)$. The symmetry factor, $S_{F}$, equals $\frac{1}{2}$ for the production of two identical Higgs bosons, and is 1 otherwise.

The Feynman diagrams contributing to the $g g \rightarrow h h, H H$, $h H$, and $A A$ processes are presented in Fig. 1, while the contributions to the processes $g g \rightarrow h A$ and $H A$ are shown in Fig. 2. We now list the resulting helicity amplitudes for these two classes of processes, starting with the case where both
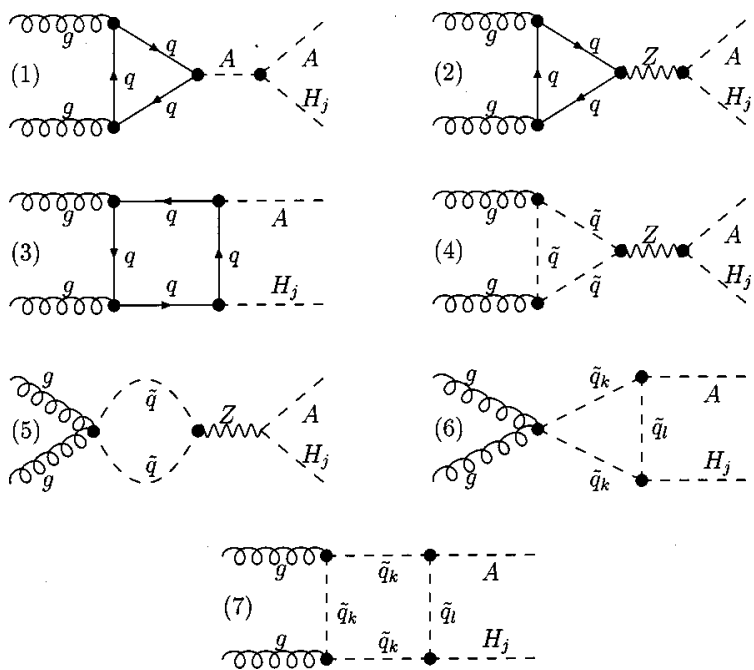

FIG. 2. Feynman diagrams for the $h A$ and $H A$ Higgs boson pair production. $H_{j}=h, H$ for $j=1,2$, respectively, $\tilde{q}_{k(l)}=\tilde{q}_{1}, \tilde{q}_{2}$ for $k(l)=1,2$. The crossed diagrams are not shown. 
produced Higgs boson have the same $C P$ properties. We have used the FeynCalc package [22] for the analytical calculation.

\section{A. Invariant amplitudes for $g g \rightarrow h h, H H, h H$, and $A A$}

\section{Quark loop contributions}

By assumption these processes conserve $C P$, which implies that $M_{+_{+}}=M_{--}$and $M_{+_{-}}=M_{-_{+}}$. The two independent helicity amplitudes for the production of two $C P$-even Higgs bosons $H_{i} H_{j}(i, j=1,2$ for $h$ and $H$, respectively), where the superscript on the amplitudes refers to the number of the Feynman diagram in Fig. 1, are as follows:

$$
\begin{aligned}
& M_{++}^{(1)}\left(H_{i} H_{j}\right)=\frac{-i \alpha_{s} m_{q} V_{H_{k} q q} V_{H_{i} H_{j} H_{k}}}{\pi\left[\left(\hat{s}-m_{H_{k}}^{2}\right)+i m_{H_{k}} \Gamma_{H_{k}}\right]}\left[2+\left(4 m_{q}^{2}-\hat{s}\right) C(0,0, \hat{s})\right] \\
& M_{+-}^{(1)}\left(H_{i} H_{j}\right)=0 \text {; } \\
& M_{++}^{(2)}\left(H_{i} H_{j}\right)=\frac{i \alpha_{s}}{2 \pi \hat{s}} V_{H_{i} q q} V_{H_{j} q q}\left\{-4 \hat{s}-8 m_{q}^{2} C(0,0, \hat{s}) \hat{s}-\left(8 m_{q}^{2}-m_{H_{i}}^{2}-m_{H_{j}}^{2}\right)\left[T_{i} C\left(m_{H_{i}}^{2}, 0, \hat{t}\right)+U_{i} C\left(m_{H_{i}}^{2}, 0, \hat{u}\right)+T_{j} C\left(m_{H_{j}}^{2}, 0, \hat{t}\right)\right.\right. \\
& \left.+U_{j} C\left(m_{H_{j}}^{2}, 0, \hat{u}\right)-\left(m_{H_{j}}^{2} m_{H_{j}}^{2}-\hat{t} \hat{u}\right) D\left(m_{H_{i}}^{2}, 0, m_{H_{j}}^{2}, 0, \hat{t}, \hat{u}\right)\right]-2 m_{q}^{2}\left(8 m_{q}^{2}-m_{H_{i}}^{2}-m_{H_{j}}^{2}-\hat{s}\right) \hat{s}\left[D\left(m_{H_{i}}^{2}, 0, m_{H_{j}}^{2}, 0, \hat{t}, \hat{u}\right)\right. \\
& \left.\left.+D\left(m_{H_{i}}^{2}, m_{H_{j}}^{2}, 0,0, \hat{s}, \hat{t}\right)+D\left(m_{H_{i}}^{2}, m_{H_{j}}^{2}, 0,0, \hat{s}, \hat{u}\right)\right]\right\} \\
& M_{+-}^{(2)}\left(H_{i} H_{j}\right)=\frac{-i \alpha_{s} V_{H_{i} q q} V_{H_{j} q q}}{2 \pi\left(m_{H_{i}}^{2} m_{H_{j}}^{2}-\hat{t} \hat{u}\right)}\left\{\left(8 m_{q}^{2}-\hat{t}-\hat{u}\right)\left(2 m_{H_{i}}^{2} m_{H_{j}}^{2}-\hat{t}^{2}-\hat{u}^{2}\right) C\left(m_{H_{i}}^{2}, m_{H_{j}}^{2}, \hat{s}\right)+\left(m_{H_{i}}^{2} m_{H_{j}}^{2}-8 m_{q}^{2} \hat{t}+\hat{t}^{2}\right)\left[T_{i} C\left(m_{H_{i}}^{2}, 0, \hat{t}\right)\right.\right. \\
& \left.+T_{j} C\left(m_{H_{j}}^{2}, 0, \hat{t}\right)-\hat{s} C(0,0, \hat{s})+\hat{s} \hat{t} D\left(m_{H_{i}}^{2}, m_{H_{j}}^{2}, 0,0, \hat{s}, \hat{t}\right)\right]+\left(m_{H_{i}}^{2} m_{H_{j}}^{2}-8 m_{q}^{2} \hat{u}+\hat{u}^{2}\right)\left[U_{i} C\left(m_{H_{i}}^{2}, 0, \hat{u}\right)\right. \\
& \left.+U_{j} C\left(m_{H_{j}}^{2}, 0, \hat{u}\right)-\hat{s} C(0,0, \hat{s})+\hat{s} \hat{u} D\left(m_{H_{i}}^{2}, m_{H_{j}}^{2}, 0,0, \hat{s}, \hat{u}\right)\right]+2 m_{q}^{2}\left(m_{H_{i}}^{2} m_{H_{j}}^{2}-\hat{t} \hat{u}\right)\left(8 m_{q}^{2}-\hat{t}-\hat{u}\right) \\
& \left.\times\left[D\left(m_{H_{i}}^{2}, 0, m_{H_{j}}^{2}, 0, \hat{t}, \hat{u}\right)+D\left(m_{H_{i}}^{2}, m_{H_{j}}^{2}, 0,0, \hat{s}, \hat{t}\right)+D\left(m_{H_{i}}^{2}, m_{H_{j}}^{2}, 0,0, \hat{s}, \hat{u}\right)\right]\right\} .
\end{aligned}
$$

Here, $V_{H_{k} q q}$ and $V_{H_{i} H_{j} H_{k}}$ are the Yukawa coupling constants of $H_{k}$ to quarks and the trilinear Higgs couplings, respectively. Expressions for these couplings in the MSSM are given in Appendix A while we list in Appendix B our choice for the polarization vectors. We have also defined the quantities

$$
T_{i}=\left(m_{H_{i}}^{2}-\hat{t}\right) \quad \text { and } \quad U_{i}=\left(m_{H_{i}}^{2}-\hat{u}\right) .
$$

Furthermore, the loop integrals appearing in Eqs. (3) are defined in terms of the Passarino-Veltman scalar functions $C_{0}$ and $D_{0}$ [23] (see Appendix B for our conventions) as

$$
\begin{gathered}
C(a, b, c)=C_{0}\left(a, b, c, m_{q}, m_{q}, m_{q}\right) ; \\
D(a, b, c, x, y, z)=D_{0}\left(a, b, c, x, y, z, m_{q}, m_{q}, m_{q}, m_{q}\right) .
\end{gathered}
$$

The scattering amplitudes for the production of a pair of pseudo-scalar Higgs boson are

$$
\begin{gathered}
M_{++}^{(1)}(A A)=M_{++}^{(1)}\left(H_{i} H_{j}\right)\left[V_{H_{i} H_{j} H_{j}} \rightarrow V_{H_{i} A A}\right] \\
M_{+-}^{(1)}(A A)=0 ; \\
M_{++}^{(2)}(A A)=\frac{i \alpha_{s}}{\pi \hat{s}} V_{A q q} V_{A q q}\left\{2 \hat{s}+4 m_{q}^{2} C(0,0, \hat{s}) \hat{s}-2 m_{A}^{2}\left[T_{A} C\left(m_{A}^{2}, 0, \hat{t}\right)+U_{A} C\left(m_{A}^{2}, 0, \hat{u}\right)\right]\right. \\
+m_{A}^{2}\left(m_{A}^{4}-\hat{u} \hat{t}\right) D\left(m_{A}^{2}, 0, m_{A}^{2}, 0, \hat{t}, \hat{u}\right)-m_{q}^{2} \hat{s}(\hat{t}+\hat{u}) \\
\left.\times\left[D\left(m_{A}^{2}, 0, m_{A}^{2}, 0, \hat{t}, \hat{u}\right)+D\left(m_{A}^{2}, m_{A}^{2}, 0,0, \hat{s}, \hat{t}\right)+D\left(m_{A}^{2}, m_{A}^{2}, 0,0, \hat{s}, \hat{u}\right)\right]\right\} ;
\end{gathered}
$$




$$
\begin{aligned}
M_{+-}^{(2)}(A A)= & \frac{-i \alpha_{s}}{2 \pi\left(m_{A}^{4}-\hat{t} \hat{u}\right)} V_{A q q} V_{A q q}\left\{\hat{s}\left(2 m_{A}^{4}+\hat{t}^{2}+\hat{u}^{2}\right) C(0,0, \hat{s})-2 T_{A}\left(m_{A}^{4}+\hat{t}^{2}\right) C\left(m_{A}^{2}, 0, \hat{t}\right)\right. \\
& -2 U_{A}\left(m_{A}^{4}+\hat{u}^{2}\right) C\left(m_{A}^{2}, 0, \hat{u}\right)+(\hat{t}+\hat{u})\left(2 m_{A}^{4}-\hat{t}^{2}-\hat{u}^{2}\right) C\left(m_{A}^{2}, m_{A}^{2}, \hat{s}\right)+2 m_{q}^{2}(\hat{t}+\hat{u})\left(m_{A}^{4}-\hat{t} \hat{u}\right) \\
& \times\left[D\left(m_{A}^{2}, 0, m_{A}^{2}, 0, \hat{t}, \hat{u}\right)+D\left(m_{A}^{2}, m_{A}^{2}, 0,0, \hat{s}, \hat{t}\right)+D\left(m_{A}^{2}, m_{A}^{2}, 0,0, \hat{s}, \hat{u}\right)\right] \\
& \left.-\hat{s}\left[\hat{t}\left(m_{A}^{4}+\hat{t}^{2}\right) D\left(m_{A}^{2}, m_{A}^{2}, 0,0, \hat{s}, \hat{t}\right)+\hat{u}\left(m_{A}^{4}+\hat{u}^{2}\right) D\left(m_{A}^{2}, m_{A}^{2}, 0,0, \hat{s}, \hat{u}\right)\right]\right\}
\end{aligned}
$$

Analogously to the production of $C P$-even Higgs bosons, we have defined the quantities

$$
T_{A}=\left(m_{A}^{2}-\hat{t}\right) \quad \text { and } \quad U_{A}=\left(m_{A}^{2}-\hat{u}\right)
$$

As a check of our calculations we verified that our results for the quark (squark) loop contributions to the Higgs pair production are invariant under QCD gauge transformations. Furthermore, our results agree with those of Ref. [20].

\section{Squark loop contributions}

We now turn to the new results of this paper, i.e., the squark loop contributions depicted in diagrams (3)-(8) in Fig. 1. These can be grouped into three sets of diagrams, $(3)+(4),(5)+(6)$, and $(7)+(8)$, which are gauge invariant and finite. Moreover, diagrams (7) and (8) are finite by themselves and we therefore list their contributions separately, treating QCD interactions in the 't Hooft-Feynman gauge:

$$
\begin{aligned}
& M_{++}^{(3+4)}\left(H_{i} H_{j}\right)=\frac{i \alpha_{s} V_{H_{l}} \tilde{q}_{k} \tilde{q}_{k} V_{H_{l} H_{i} H_{j}}}{2 \pi\left[\left(\hat{s}-m_{H_{l}}^{2}\right)+i m_{H_{l}} \Gamma_{H_{l}}\right]}\left[1+2 m_{\tilde{q}_{k}}^{2} C_{k k k}(0,0, \hat{s})\right] \\
& M_{+-}^{(3+4)}\left(H_{i} H_{j}\right)=0 \\
& M_{++}^{(5+6)}\left(H_{i} H_{j}\right)=\frac{-i \alpha_{s}}{2 \pi} V_{H_{i} H_{j}} \tilde{q}_{k} \tilde{q}_{k}\left[1+2 m_{\tilde{q}_{k}}^{2} C_{k k k}(0,0, \hat{s})\right] ; \\
& M_{+-}^{(5+6)}\left(H_{i} H_{j}\right)=0 \\
& M_{++}^{(7)}\left(H_{i} H_{j}\right)=\frac{-i \alpha_{s}}{\pi} V_{H_{i} \tilde{q}_{k} \tilde{q}_{l}} V_{H_{j} \tilde{q}_{k} \tilde{q}_{l}} C_{k l k}\left(m_{H_{i}}^{2}, m_{H_{j}}^{2}, \hat{s}\right) \\
& M_{+-}^{(7)}\left(H_{i} H_{j}\right)=0 \\
& M_{++}^{(8)}\left(H_{i} H_{j}\right)=\frac{i \alpha}{2 \pi \hat{s}} V_{H_{i}} \tilde{q}_{k} \tilde{q}_{l} V_{H_{j}} \tilde{q}_{k} \tilde{q}_{l}\left\{T_{i} C_{l k k}\left(m_{H_{i}}^{2}, 0, \hat{t}\right)+U_{i} C_{k l l}\left(m_{H_{i}}^{2}, 0, \hat{u}\right)+T_{j} C_{k l l}\left(m_{H_{j}}^{2}, 0, \hat{t}\right)+U_{j} C_{l k k}\left(m_{H_{j}}^{2}, 0, \hat{u}\right)\right. \\
& +2 \hat{s} C_{k l k}\left(m_{H_{i}}^{2}, m_{H_{j}}^{2}, \hat{s}\right)+\left[\left(m_{\tilde{q}_{l}}^{2}-m_{\tilde{q}_{k}}^{2}\right) \hat{s}-\left(m_{H_{i}}^{2} m_{H_{j}}^{2}-\hat{t} \hat{u}\right)\right] D_{l k k l}\left(m_{H_{i}}^{2}, 0, m_{H_{j}}^{2}, 0, \hat{t}, \hat{u}\right) \\
& \left.+2 \hat{s} m_{\tilde{q}_{k}}^{2}\left[D_{l k k l}\left(m_{H_{i}}^{2}, 0, m_{H_{j}}^{2}, 0, \hat{t}, \hat{u}\right)+D_{k l k k}\left(m_{H_{i}}^{2}, m_{H_{j}}^{2}, 0,0, \hat{s}, \hat{t}\right)+D_{k l k k}\left(m_{H_{i}}^{2}, m_{H_{j}}^{2}, 0,0, \hat{s}, \hat{u}\right)\right]\right\} \\
& M_{+-}^{(8)}\left(H_{i} H_{j}\right)=\frac{i \alpha_{s}}{2 \pi\left(m_{H_{i}}^{2} m_{H_{j}}^{2}-\hat{t} \hat{u}\right)} V_{H_{i} \tilde{q}_{k} \tilde{q}_{l}} V_{H_{j} \tilde{q}_{k} \tilde{q}_{l}}\left\{\hat{s}\left(2 m_{\tilde{q}_{k}}^{2}-2 m_{\tilde{q}_{l}}^{2}+\hat{t}+\hat{u}\right) C_{k k k}(0,0, \hat{s})-\hat{t}\left[T_{i} C_{l k k}\left(m_{H_{i}}^{2}, 0, \hat{t}\right)+T_{j} C_{l k k}\left(m_{H_{j}}^{2}, 0, \hat{t}\right)\right]\right. \\
& -\hat{u}\left[U_{i} C_{l k k}\left(m_{H_{i}}^{2}, 0, \hat{u}\right)+U_{j} C_{l k k}\left(m_{H_{j}}^{2}, 0, \hat{u}\right)\right]-T_{j}\left(m_{\tilde{q}_{k}}^{2}-m_{\tilde{q}_{l}}^{2}\right)\left[C_{k l l}\left(m_{H_{j}}^{2}, 0, \hat{t}\right)+C_{l k k}\left(m_{H_{j}}^{2}, 0, \hat{t}\right)\right]-U_{i}\left(m_{\tilde{q}_{k}}^{2}-m_{\tilde{q}_{l}}^{2}\right) \\
& \times\left[C_{k l l}\left(m_{H_{i}}^{2}, 0, \hat{u}\right)+C_{l k k}\left(m_{H_{i}}^{2}, 0, \hat{u}\right)\right]+\left(2 m_{H_{i}}^{2} m_{H_{j}}^{2}-\hat{t}^{2}-\hat{u}^{2}\right) C_{k l k}\left(m_{H_{i}}^{2}, m_{H_{j}}^{2}, \hat{s}\right)
\end{aligned}
$$




$$
\begin{aligned}
& +\left[-\hat{s}\left(m_{\tilde{q}_{k}}^{2}-m_{\tilde{q}_{l}}^{2}\right)^{2}+\left(m_{\tilde{q}_{k}}^{2}+m_{\tilde{q}_{l}}^{2}\right)\left(m_{H_{i}}^{2} m_{H_{j}}^{2}-\hat{t} \hat{u}\right)\right]\left[D_{l k k l}\left(m_{H_{i}}^{2}, 0, m_{H_{j}}^{2}, 0, \hat{t}, \hat{u}\right)+D_{k l k k}\left(m_{H_{i}}^{2}, m_{H_{j}}^{2}, 0,0, \hat{s}, \hat{t}\right)\right. \\
& \left.+D_{k l k k}\left(m_{H_{i}}^{2}, m_{H_{j}}^{2}, 0,0, \hat{s}, \hat{u}\right)\right]+\left[-\hat{s} \hat{t}^{2}-\left(m_{\tilde{q}_{k}}^{2}-m_{\tilde{q}_{l}}^{2}\right)\left(2 \hat{t} \hat{s}-\left(m_{H_{i}}^{2} m_{H_{j}}^{2}-\hat{t} \hat{u}\right)\right)\right] D_{k l k k}\left(m_{H_{i}}^{2}, m_{H_{j}}^{2}, 0,0, \hat{s}, \hat{t}\right) \\
& \left.+\left[-\hat{s} \hat{u}^{2}-\left(m_{\tilde{q}_{k}}^{2}-m_{\tilde{q}_{l}}^{2}\right)\left(2 \hat{u} \hat{s}-\left(m_{H_{i}}^{2} m_{H_{j}}^{2}-\hat{t} \hat{u}\right)\right)\right] D_{k l k k}\left(m_{H_{i}}^{2}, m_{H_{j}}^{2}, 0,0, \hat{s}, \hat{u}\right)\right\} .
\end{aligned}
$$

MSSM predictions for the trilinear Higgs-squark-squark couplings $V_{H_{l}} \tilde{q}_{k} \tilde{q}_{k}$ and the quartic Higgs-Higgs-squark-squark couplings $V_{H_{i} H_{j}} \tilde{q}_{k} \tilde{q}_{k}$ are given in Appendix A; note that we need the former two couplings only for two identical squarks. The loop functions appearing in Eqs. (8) depend on the squark masses and are defined as

$$
\begin{gathered}
C_{i j k}(a, b, c)=C_{0}\left(a, b, c, m_{\tilde{q}_{i}}, m_{\tilde{q}_{j}}, m_{\tilde{q}_{k}}\right) \\
D_{i j k l}(a, b, c, x, y, z)=D_{0}\left(a, b, c, x, y, z, m_{\tilde{q}_{i}}, m_{\tilde{q}_{j}}, m_{\tilde{q}_{k}}, m_{\tilde{q}_{l}}\right) .
\end{gathered}
$$

The corresponding expressions for a pair of pseudo-scalar Higgs bosons are

$$
\begin{gathered}
M_{++}^{(3+4)}(A A)=M_{++}^{(3+4)}\left(H_{i} H_{j}\right)\left[V_{H_{l} H_{i} H_{j}} \rightarrow V_{H_{l} A A}\right] \\
M_{+-}^{(3+4)}(A A)=0 ; \\
M_{++}^{(5+6)}(A A)=M_{++}^{(5+6)}\left(H_{i} H_{j}\right)\left[V_{H_{i} H_{j}} \tilde{q}_{k} \tilde{q}_{k} \rightarrow V_{A A \tilde{q}_{k}} \tilde{q}_{k}\right] \\
M_{+-}^{(5+6)}(A A)=0 ; \\
M_{++}^{(7)}(A A)=M_{++}^{(7)}\left(H_{i} H_{j}\right)\left[V_{H_{(i, j)}} \tilde{q}_{k} \tilde{q}_{k} \rightarrow V_{\left.A \tilde{q}_{k} \tilde{q}_{l}\right]}^{(7)}(A A)=0 ;\right. \\
M_{++}^{(8)}(A A)=-M_{++}^{(8)}\left(H_{i} H_{j}\right)\left[V_{H_{(i, j)}} \tilde{q}_{k} \tilde{q}_{l} \rightarrow V_{A} \tilde{q}_{k} \tilde{q}_{l}\right] \\
M_{+-}^{(8)}(A A)=-M_{+-}^{(8)}\left(H_{i} H_{j}\right)\left[V_{H_{(i, j)}} \tilde{q}_{k} \tilde{q}_{l} \rightarrow V_{A \tilde{q}_{k}} \tilde{q}_{l}\right]
\end{gathered}
$$

\section{B. Invariant amplitudes for $g g \rightarrow A h, \quad A H$}

We now turn to the production of two Higgs bosons with different $C P$ quantum numbers which only receive contributions from quark loops. Since we assumed $C P$ invariance in the Higgs boson and squark sectors, the squark contributions to the production of a $C P$-even and a $C P$-odd Higgs boson, i.e., the diagrams (4), (5), (6), and (7) of Fig. 2 add to zero. Let us have a closer look at this. Note that $A$ only couples to two different squark mass eigenstates; this immediately eliminates the equivalent of diagrams (5) and (6) in Fig. 1. Moreover, $V_{A \tilde{q}_{1} \tilde{q}_{2}}=-V_{A \tilde{q}_{2} \tilde{q}_{1}}$, and consequently the two possible orientations of the loop momentum in diagrams (6) and (7) in Fig. 2 exactly cancel each other. Finally a same-flavor $\tilde{q} \widetilde{q}^{*}$ pair in a color-singlet state coupling to a $Z$ boson is in a $C P$-even state, while one $C P$-even and one $C P$-odd Higgs boson coupling to a $Z$ boson are in a $C P$-odd state; this explains why diagrams (4) and (5) vanish. For completeness we give expressions for the quark loop contribution in our notation; our results agree with those of Ref. [20]. Note that we again only have two independent helicity amplitudes since $M_{++}=-M_{--}$and $M_{+-}=-M_{-+}$for $A h$ and $A H$ production.

$$
\begin{aligned}
M_{++}^{(1)}\left(A H_{i}\right)= & \frac{i \alpha_{s} m_{q}}{\pi\left[\left(\hat{s}-m_{A}^{2}\right)+i m_{A} \Gamma_{A}\right]} V_{A q q} V_{H_{j} A A} \hat{s} C(0,0, \hat{s}) ; \\
M_{++}^{(2)}\left(A H_{i}\right)= & \frac{-i \alpha_{s} g_{Z} T_{3}^{q}\left(m_{H_{i}}^{2}-m_{A}^{2}\right)\left(\hat{s}-M_{Z}^{2}\right)}{2 \pi m_{Z}^{2}\left[\left(\hat{s}-m_{Z}^{2}\right)+i m_{Z} \Gamma_{Z}\right]} \\
& \times V_{Z A H_{i}}\left[1+2 m_{q}^{2} C(0,0, \hat{s})\right] ; \\
M_{++}^{(3)}\left(A H_{i}\right)= & \frac{i \alpha_{s}}{2 \pi \hat{s}} V_{H_{i} q q} V_{A q q}\left\{( m _ { A } ^ { 2 } - m _ { H _ { i } } ^ { 2 } ) \left[T_{A} C\left(m_{A}^{2}, 0, \hat{t}\right)\right.\right. \\
& +T_{i} C\left(m_{H_{i}}^{2}, 0, \hat{t}\right)+U_{A} C\left(m_{A}^{2}, 0, \hat{u}\right) \\
& +U_{i} C\left(m_{H_{i}}^{2}, 0, \hat{u}\right)-\left(m_{A}^{2} m_{H_{i}}^{2}-\hat{t} \hat{u}\right) \\
& \left.\times D\left(m_{A}^{2}, 0, m_{H_{i}}^{2}, 0, \hat{t}, \hat{u}\right)\right]+2 m_{q}^{2} \hat{s}\left(T_{A}+U_{A}\right) \\
& \times\left[D\left(m_{A}^{2}, 0, m_{H_{i}}^{2}, 0, \hat{t}, \hat{u}\right)+D\left(m_{A}^{2}, m_{H_{i}}^{2}, 0,0, \hat{s}, \hat{t}\right)\right. \\
& \left.\left.+D\left(m_{A}^{2}, m_{H_{i}}^{2}, 0,0, \hat{s}, \hat{u}\right)\right]\right\} ;
\end{aligned}
$$

$$
\begin{aligned}
M_{+-}^{(3)}\left(A H_{i}\right) & \\
= & \frac{-i \alpha_{s}}{2 \pi\left(m_{A}^{2} m_{H_{i}}^{2}-\hat{t} \hat{u}\right)} V_{H_{i} q q} V_{A q q}\left\{\hat{s}\left(\hat{t}^{2}-\hat{u}^{2}\right) C(0,0, \hat{s})\right. \\
& +\left(4 m_{A}^{2} m_{H_{i}}^{2}-(\hat{t}+\hat{u})^{2}\right)(\hat{t}-\hat{u}) C\left(m_{A}^{2}, m_{H_{i}}^{2}, \hat{s}\right) \\
& +\left(m_{A}^{2} m_{H_{i}}^{2}-\hat{t}^{2}\right)\left[T_{A} C\left(m_{A}^{2}, 0, \hat{t}\right)+T_{i} C\left(m_{H_{i}}^{2}, 0, \hat{t}\right)\right. \\
& \left.+\hat{s} \hat{t} D\left(m_{A}^{2}, m_{H_{i}}^{2}, 0,0, \hat{s}, \hat{t}\right)\right]-\left(m_{A}^{2} m_{H_{i}}^{2}-\hat{u}^{2}\right)
\end{aligned}
$$




$$
\begin{aligned}
& \times\left[U_{A} C\left(m_{A}^{2}, 0, \hat{u}\right)\right. \\
& \left.+U_{i} C\left(m_{H_{i}}^{2}, 0, \hat{u}\right)+\hat{s} \hat{u} D\left(m_{A}^{2}, m_{H_{i}}^{2}, 0,0, \hat{s}, \hat{u}\right)\right]+2 m_{q}^{2}(\hat{t}-\hat{u}) \\
& \times\left(m_{A}^{2} m_{H_{i}}^{2}-\hat{t} \hat{u}\right)\left[D\left(m_{A}^{2}, 0, m_{H_{i}}^{2}, 0, \hat{t}, \hat{u}\right)\right. \\
& \left.\left.+D\left(m_{A}^{2}, m_{H_{i}}^{2}, 0,0, \hat{s}, \hat{t}\right)+D\left(m_{A}^{2}, m_{H_{i}}^{2}, 0,0, \hat{s}, \hat{u}\right)\right]\right\},
\end{aligned}
$$

where $T_{3}^{q}$ is the third component of the weak isospin of the quark running in the loop.

\section{NUMERICAL RESULTS}

We are now ready to illustrate the importance of squark loop contributions with a few examples. For the numerical analysis we have used the leading order CTEQ4L parametrization of the parton distribution function of the proton [24], choosing the QCD renormalization and factorization scales to be the averaged sum of the masses of the Higgs bosons in the final state. The effect of the running mass of the bottom quark can be very important, therefore, we have also included it in our calculations. In fact, when the main contribution to the processes comes from bottom-quark loops and/or bottom-squark loops, the cross section is proportional to the $\phi b \bar{b}$ Yukawa coupling to the fourth power. Taking 3 and $5 \mathrm{GeV}$ for typical running and pole $b$-quark mass respectively we can see that this effect can reduce the Higgs pair production by a factor $(3 / 5)^{4} \simeq 1 / 8$.

As discussed in Sec. II A 2, the squark loop diagrams shown in Fig. 1 fall into three groups of diagrams, (3) $+(4),(5)+(6)$, and $(7)+(8)$, the sum of diagrams in each group being finite and gauge invariant. In unpolarized $p p$ or $p \bar{p}$ scattering, where only the sum of the square of all helicity amplitudes is measurable, the squark contribution can therefore be characterized by three loop functions and the associated products of coupling constants. In order to assess the importance of these three sets of diagrams, we show in Fig. 3 their individual contributions to the subprocess cross section $(\hat{\sigma})$ for the production of $h h$ pairs. For the sake of simplicity, only a single squark mass eigenstate $\left(\tilde{b}_{1}\right)$ was included here, whose mass is given on the $x$ axis. We chose $m_{h}=100 \mathrm{GeV}$ and $\sqrt{\hat{s}}=3 m_{h}=300 \mathrm{GeV}$ as typical values. Note that diagrams (3), (4), (7) and (8) involve dimensionful couplings, while in diagrams (5) and (6) only dimensionless couplings appear. In order to show the corresponding loop functions, we have therefore considered fixed 'typical' coupling constants $\left(\tan \beta=50, \quad M_{\tilde{q}}=325 \mathrm{GeV}, \quad M_{A}\right.$ $\left.=100 \mathrm{GeV}, A_{t}=A_{b}=-410 \mathrm{GeV}, \mu=-640 \mathrm{GeV}\right)$. However, these couplings were not varied as the mass of the squark in the loop is changed, while physical couplings do usually depend on the masses of the squark mass eigenstates, e.g., through the change of the $\tilde{q}_{L}-\tilde{q}_{R}$ mixing angle; see Appendix A.

The loop function describing diagrams (3) + (4) is given in Eqs. (8a) and (8b). It is the same (up to an overall factor) as that describing the squark loop contribution to single Higgs boson production [6]. This contribution (dotted curve)

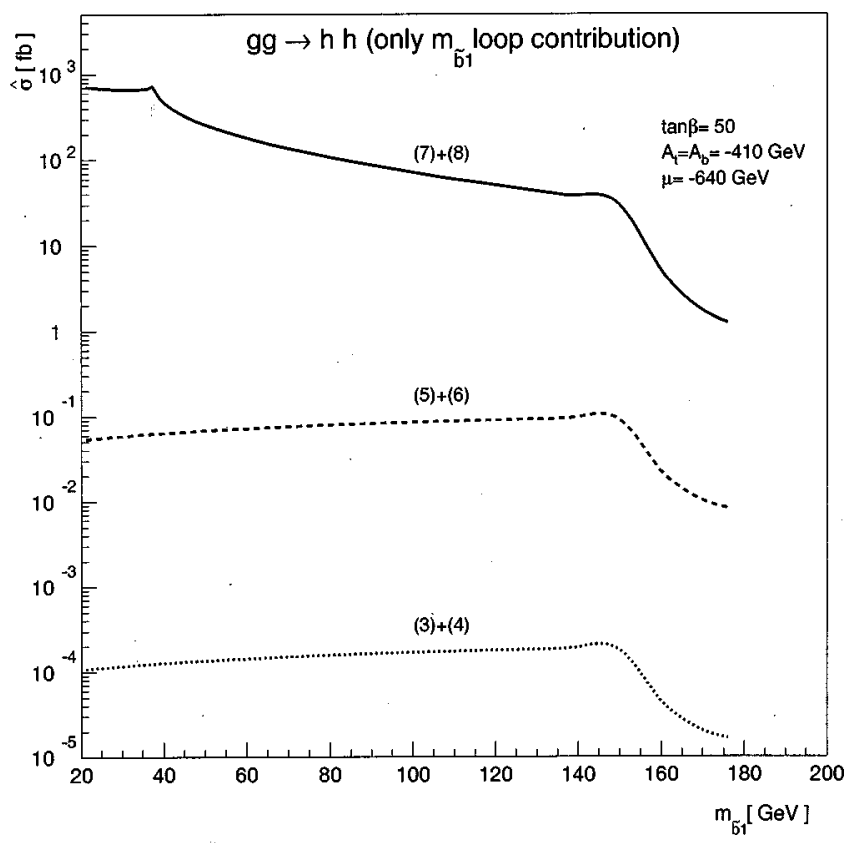

FIG. 3. Contributions of the $(3)+(4),(5)+(6)$, and (7) $+(8)$ diagram sets to the subprocess cross section for the production of $h h$ pairs as a function of $m_{\tilde{b}_{1}}$. We included only the $\widetilde{b}_{1}$ effects and assumed $m_{h}=100 \mathrm{GeV}$ and $\sqrt{\hat{s}}=300 \mathrm{GeV}$.

seems to be important only when real $H \rightarrow h h$ decays are possible and $\tan \beta$ is not too large, since otherwise $B(H$ $\rightarrow h h$ ) becomes very small. The contribution from diagrams $(5)+(6)$ (dashed curve), which involves quartic scalar couplings, is given in Eqs. (8c) and (8d). We find that this contribution can increase the total cross section by no more than a factor of a few. The reason is that this quartic scalar coupling cannot significantly exceed the square of the corresponding Yukawa coupling appearing in the quark loop contribution, and the squarks in the loop cannot be much lighter than the corresponding quarks. Of course, $m_{\tilde{b}_{1}} \gg m_{b}$, but $m_{\tilde{t}_{1}}<m_{t}$ is still allowed.

The potentially largest contribution therefore comes from diagrams $(7)+(8)$ (solid line), Eqs. (8e)-(8h), which involve trilinear Higgs-squark-squark couplings. These dimensionful couplings depend on unknown soft breaking parameters, and might be (much) larger than the mass of the lighter squark in the loop. On the other hand, while the loop functions for diagrams $(3)+(4)$ and $(5)+(6)$ slightly increase with increasing squark mass as long as $m_{\tilde{q}}<\sqrt{\hat{s}} / 2$, the loop function for diagrams $(7)+(8)$ starts to decrease as soon as $m_{\tilde{q}}>m_{h} / 2$ [25]. Once $m_{\tilde{q}}>\sqrt{\hat{s}} / 2$, all squark loop functions become real, and drop rapidly with increasing $m_{\tilde{q}}$, approximately like $m_{\tilde{q}}^{-4}$. Because of the quick falling parton distribution functions, the largest contribution to the total Higgs pair production cross section come from values of $\hat{s}$ not far above threshold. Figure 3 then shows that squark loop contributions to the total cross section can only be large if the mass of the squarks in the loop does not much exceed that of the produced Higgs bosons.

The results of Fig. 3 allowed us to search the MSSM 

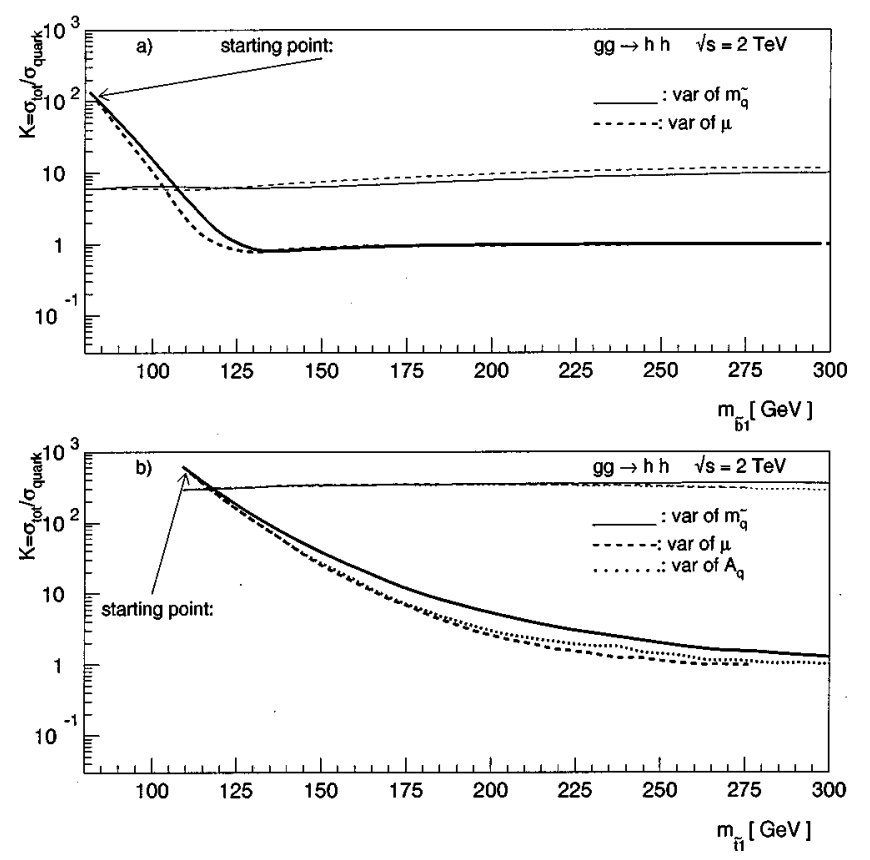

FIG. 4. Ratio of the total cross section to the cross section including only the quark contributions for $g g \rightarrow h h$ at the Tevatron. (a) corresponds to a large $\tan \beta(=50)$ scenario and we took $\left(m_{\tilde{q}}, A_{q}, \mu\right)=(325,-410,-640) \mathrm{GeV}$. The heavy solid and dashed curves have been obtained by varying, one at a time, $m_{q}$ and $\mu$, respectively. In (b) we display a low $\tan \beta \quad(=2)$ scenario using $\left(m_{\tilde{q}}, A_{q}, \mu\right)=(380,510,-975) \mathrm{GeV}$, and conventions as in (a). Here, the heavy dotted line was obtained by varying $A_{q}$. In both (a) and (b), the thin lines correspond to the enhancement needed for the total cross section to be at the level of $50 \mathrm{fb}$.

parameter space for parameters that maximize some subset of the diagrams listed in Fig. 1. In these searches we imposed the following constraints on parameters. First, most SUSY models predict

$$
1<\tan \beta \leqslant \frac{m_{t}\left(m_{t}\right)}{m_{b}\left(m_{t}\right)} \simeq 55 .
$$

Second, we have interpreted the unsuccessful search for Higgs bosons at LEP [26] to imply $m_{h} \geqslant 90 \mathrm{GeV}$ if the $Z Z h$ coupling has similar strength as the corresponding coupling in the SM. Otherwise the $Z A h$ coupling is large, and $\left(m_{A}\right.$ $\left.+m_{h}\right) \geqslant 175 \mathrm{GeV}$ is required. When computing the masses and couplings of the $C P$-even Higgs bosons, we have included squark and quark loop corrections [27] as given by the 1-loop effective potential [28-30]. Turning to the squark sector, for simplicity we took the same soft breaking mass $m_{\tilde{q}}$ for $m_{\tilde{t}_{L}} \equiv m_{\tilde{b}_{L}}, \quad m_{\tilde{t}_{R}}$ and $m_{\tilde{b}_{R}}$, and also took the same value $A_{q}$ for the soft breaking parameters $A_{t}$ and $A_{b}$. The squark sector is then completely determined by $m_{\tilde{q}}, \quad A_{q}, \quad \tan \beta$ and the supersymmetric Higgs mass parameter $\mu$. In our scans we have imposed the LEP search limit [31] $m_{\tilde{t}_{1}}, \quad m_{\tilde{b}_{1}} \geqslant 80 \mathrm{GeV}$ [32].

As anticipated, we found that the potentially largest squark loop contribution comes from diagrams $(7)+(8)$. In Figs. 4(a) and 4(b) we show that (mostly) due to this contri- bution, squark loops can increase the total $h$-pair cross section at the Tevatron by more than two orders of magnitude if the mass of the lighter squark eigenstate in the loop is close to its experimental lower limit. In Fig. 4(a) we chose $m_{A}$ $=100 \mathrm{GeV}$ and $\tan \beta=50$. The thick curves start at $\left(m_{\tilde{q}}, A_{q}, \mu\right)=(325,-410,-640) \mathrm{GeV}$, corresponding to $h \widetilde{b}_{1} \widetilde{b}_{1}$ coupling $V_{h \widetilde{b}_{1} \widetilde{b}_{1}}=455 \mathrm{GeV}$. This choice saturates the LEP $A h$ search limit (the $Z Z h$ coupling is very small here). The heavy solid and dashed curves have been obtained by varying $m_{\tilde{q}}$ and $\mu$, respectively, keeping all other parameters fixed. Note that increasing $\mu$ (i.e., decreasing $|\mu|$ ) not only increases $m_{\tilde{b}_{1}}$, but also reduces the $h \widetilde{b}_{1} \widetilde{b}_{1}$ coupling, leading to a rapid drop-off of the squark loop contribution. On the other hand, increasing $m_{\tilde{q}}$ leads to a somewhat slower decrease of this coupling. In both cases the squark loop contribution to the total cross section becomes essentially negligible for $m_{\tilde{b}_{1}} \geqslant 150 \mathrm{GeV}$. For slightly smaller squark masses, there is mild destructive interference between quark and squark loops.

In Fig. 4(b) we have chosen $m_{A}=500 \mathrm{GeV}$ and $\tan \beta$ $=2.0$. The thick curves originate at $\left(m_{\tilde{q}}, A_{q}, \mu\right)=(380,510$, -975) $\mathrm{GeV}$, which saturates the LEP $Z Z h$ search limit [33] and gives $V_{h \tilde{t}_{1} \tilde{t}_{1}}=475 \mathrm{GeV}$. The thick heavy solid, dashed and dotted lines have been obtained by varying, one at a time, $m_{q}, A_{q}$, and $\mu$, respectively. We see that here the squark loop contribution remains significant out to $m_{\tilde{t}_{1}}$ $\simeq 200 \mathrm{GeV}$. This is partly due to the fact that the quark loop contribution in Fig. 4(b) is about fifty times smaller than in Fig. 4(a), which in turn results from the large enhancement of the $b$-loop contribution compared to the SM, by roughly a factor $\tan ^{2} \beta=2,500$ in the amplitude. The bottom Yukawa coupling in Fig. 4(a) is nearly as large as the top Yukawa coupling in Fig. 4(b). The former than gives a much larger quark loop contribution than the latter, since for the relevant values of $\hat{s}$, the absolute value of the (mostly imaginary) $b$-quark loop function is much larger than that of the (mostly real) $t$-quark loop function.

This enhancement of the contribution of $b$-quark loops at large $\tan \beta$ also implies that prospects for detecting a signal for the production of neutral Higgs boson pairs at the next Tevatron collider run might be better if $\tan \beta$ is large. We estimate that a cross section of $50 \mathrm{fb}$ or more might be detectable. This would lead to roughly 10 events per experiment, each with 4 high- $p_{T}$ tagged $b$-jets and double $b \bar{b}$ invariant mass peaking, assuming an integrated luminosity of $2 \mathrm{fb}^{-1}$ and an overall efficiency of $10 \%$. The enhancement of the pure quark loop contribution required to achieve this cross section is given by the thin lines in Figs. 4(a) and 4(b). We see that for $\tan \beta=50$ the total cross section can exceed this sensitivity limit by more than an order of magnitude. However, the maximal light squark mass compatible with such a large $h h$ production cross section is about the same at low and at large $\tan \beta(\simeq 110 \mathrm{GeV})$.

It is interesting to notice that in Fig. $4(\mathrm{a}), m_{h}$ is significantly below $m_{A}$, i.e., the difference is up to $25 \mathrm{GeV}$, for all values of $m_{\tilde{b}_{1}}$ where squark loop contributions are signifi- 

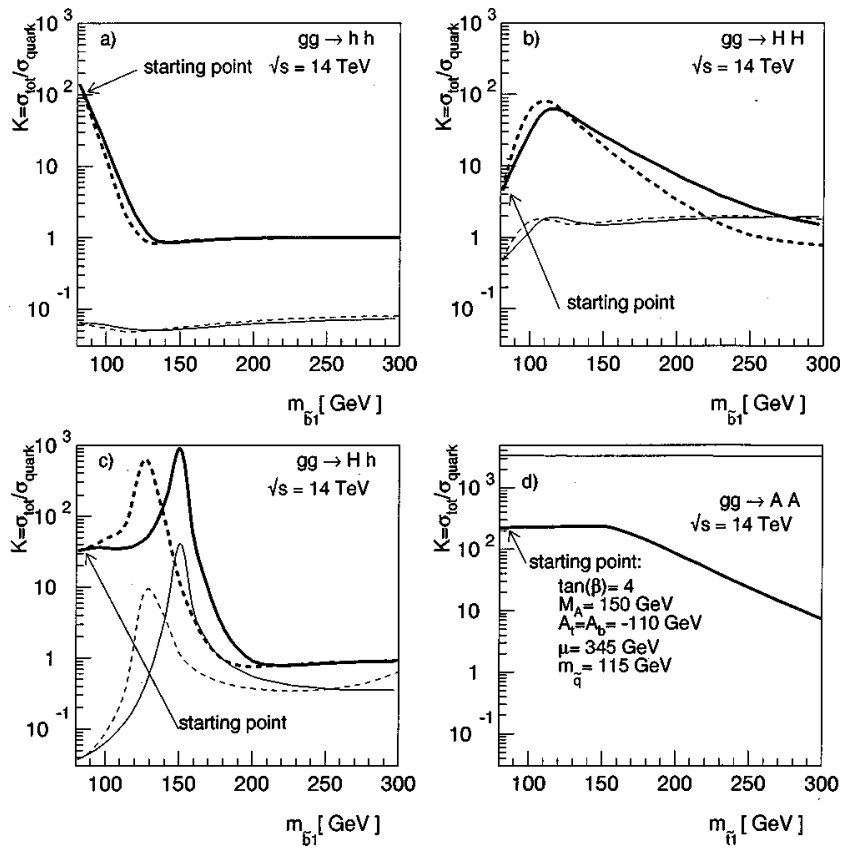

FIG. 5. Same as in Fig. 4 for the $h h$ (a), $H H$ (b), $H h$ (c) and $A A$ (d) production at the LHC. The conventions are the same as in Fig. 4. In (a)-(c) we chose the parameters used for Fig. 4(a). In (d) we assumed that $m_{A}=150 \mathrm{GeV}, \tan \beta=4, A_{q}=-110 \mathrm{GeV}, \mu$ $=345 \mathrm{GeV}$, and $m_{q}$ between 115 and $350 \mathrm{GeV}$.

cant. This mass pattern, which is quite unusual for large $\tan \beta$, is due to non-logarithmic corrections to the Higgs boson mass matrix involving trilinear scalar interactions; technically, due to large contributions to $\Delta_{12}$, in the notation of Refs. [28,29]. This means that the total cross sections for the production of pairs of other Higgs bosons are quite small at the Tevatron.

At high $\tan \beta$ the Higgs pair production can be enhanced by factors $\tan ^{4} \beta$ or $1 / \cos ^{4} \beta$ with respect to the SM production mechanism. The only exception to this is the $h h(H H)$ channel in the large (small) $M_{A}$ limit, in which the factor $\sin \alpha / \cos \beta(\cos \alpha / \cos \beta)$ appearing in Yukawa couplings goes to 1 . For instance, in the case that $\left(m_{\tilde{q}}, A_{q}, \mu\right)$ $=(1,1,1) \mathrm{TeV}, \tan \beta=50$, and $M_{A}=100 \mathrm{GeV}$ the total cross section at the Tevatron for the $h h, H H$, and $A A$ production is $3.3,0.034,3.9 \mathrm{fb}$, respectively. When $M_{A}$ is increased to $130 \mathrm{GeV}$ these cross sections change to $0.15,0.1$, and $0.7 \mathrm{fb}$.

The Higgs pair production cross sections do remain sizable at the LHC. In Figs. 5(a)-5(d) we show the squark loop contribution to $h h(\mathrm{a}), H H(\mathrm{~b}), h H(\mathrm{c})$, and $A A$ (d) productions. The parameters taken in Figs. 5(a)-5(c) are the same as in Fig. 4(a), which had been chosen to maximize the $h \widetilde{b}_{1} \widetilde{b}_{1}$ coupling. The comparison of Figs. 4(a) and 5(a) shows that the relative importance of squark loops is almost independent of the center-of-mass energy. Of course, the total cross section increases greatly when going from $\sqrt{s}$ $=2 \mathrm{TeV}$ to $14 \mathrm{TeV}$ due to the rapid increase in the gluongluon luminosity. This is illustrated by the thin lines, which again correspond to a total cross section of $50 \mathrm{fb}$. The quark (mostly $b$ ) loop contribution by itself now exceeds this cross section. Nevertheless, the background also increases when going from the Tevatron to the LHC. In the absence of a dedicated analysis of signal and background, we do not want to claim that a total cross section of $50 \mathrm{fb}$ necessarily gives a detectable signal at the LHC, in spite of its considerably higher anticipated luminosity.

Although the starting point of the curves in Figs. 5(a)5 (c) had been chosen to maximize $V_{h \widetilde{b}_{1} \tilde{b}_{1}}$, we find a very large squark loop contribution also for $H H$ production [Fig. $5(b)$. In this case the squark loop contribution at first increases with increasing $m_{\tilde{b}_{1}}$. The reason is that the $H \widetilde{b}_{1} \widetilde{b}_{1}$ coupling increases quickly, from $\sim 150 \mathrm{GeV}$ at the starting point of the curves to $\sim 350 \mathrm{GeV}$ near the maximum of the dark solid curve. In fact, this coupling keeps increasing even further as $m_{q}$ is increased, eventually reaching $\sim 450 \mathrm{GeV}$. However, this increase is overpowered by the rapid drop of the loop function once $m_{\tilde{b}_{1}}$ significantly exceeds $m_{H}$ (see Fig. 3).

The biggest relative contribution from squark loops appears in $h H$ production, Fig. 5(c), giving rise to an enhancement factor $\sim 500$ in some cases. In this region of parameter space the $H b \bar{b}$ Yukawa coupling becomes very small, due to the unusual mixing pattern of $C P$-even Higgs bosons caused by radiative corrections at large $\tan \beta$ and large $\left|A_{q}\right|$ and $|\mu|$. At the peak of the curves the $H b \bar{b}$ coupling vanishes completely, and the $h t \bar{t}$ coupling is quite small, leading to a very small total quark loop contribution (see the behavior of the thin lines). Notwithstanding, when $m_{q}^{\tilde{q}}$ or $\mu$ are increased beyond this point, the quark loop contribution reasserts itself while the squark loop contribution decreases in absolute size, leading to a steep drop of the relative importance of the squark contribution: it becomes essentially negligible for $m_{\tilde{b}_{1}} \geqslant 220 \mathrm{GeV}$. The suppression of the $H b \bar{b}$ coupling also explains why squark loop contributions to $H H$ production can remain significant up to $m_{\tilde{b}_{1}} \simeq 300 \mathrm{GeV}$ [see Fig. 5(b)]. In this case the dominant quark contribution comes from top quark loops, so squark loops remain significant unless $m_{\tilde{b}_{1}}^{2}$ $\gg m_{t}^{2}$.

For the parameters chosen in Figs. 5(a)-5(c) the squark loop contribution to $A A$ production is totally negligible. In this case diagrams (7) and (8) in Fig. 1 have to include at least one heavy squark mass eigenstate, since the diagonal $A \widetilde{b}_{1} \widetilde{b}_{1}^{*}$ and $A \widetilde{t}_{1} \tilde{t}_{1}^{*}$ couplings vanish identically. The offdiagonal $A \widetilde{b}_{1} \widetilde{b}_{2}^{*}$ coupling is actually quite large, $\sim 270 \mathrm{GeV}$ at the starting point of the curves in Fig. 5(a). However, at the same time $m_{\tilde{b}_{2}}=455 \mathrm{GeV}$, which suppresses the contributions from diagrams $(7)+(8)$ to an insignificant level.

In Fig. 5(d), we therefore show results for a scenario with relatively small $m_{\tilde{t}_{2}}: m_{A}=150 \mathrm{GeV}, \tan \beta=4, A_{q}=$ $-110 \mathrm{GeV}, \mu=345 \mathrm{GeV}$, and $m_{q}$ between 115 and 350 $\mathrm{GeV}$. The same set of parameters also yields a relatively large quartic $A A \widetilde{t}_{1} \tilde{t}_{1}^{*}$ coupling, so that diagrams $(5)+(6)$ in Fig. 1 are maximized. Since $\tan \beta$ is fairly small, the quark loop contribution is dominated by top quark loops; however, the loop function is also different for $C P$-odd Higgs bosons, 


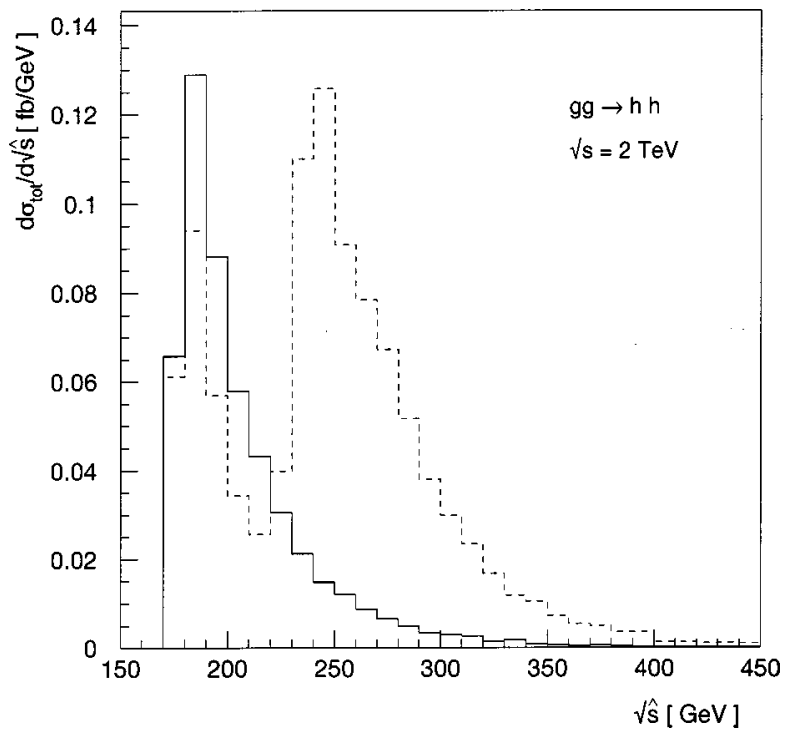

FIG. 6. $d \sigma / d \sqrt{\hat{s}}$ including only quark loops (solid line) and considering quark and squark loops (dashed line). We have chosen $\left(m_{\tilde{q}}, A_{q}, \mu\right)=(335,-410-640), M_{A}=100 \mathrm{GeV}$, and $\tan \beta=50$ for which quark and squark loops contribute almost equally: $\sigma_{\text {total }}=10 . \mathrm{fb}, \quad \sigma_{\text {squark }}=5.6 \mathrm{fb}$.

see Eqs. (6), and its contribution is suppressed by a factor $\cot ^{4} \beta \simeq 1 / 250$ compared to the SM case. Therefore diagrams $(7)+(8)$ can make a large relative contribution, as long as $m_{q} \leqslant m_{t}$. Even though squark loop contributions can increase the $A A$ cross section by a factor of about 200 in this case, it still remains well below the cross section for $\mathrm{HH}$ production. In scenarios where the $A A$ cross section is comparable to the $H H$ cross section, we find that squark loop corrections to $A A$ production are quite modest.

Given that we are only working in leading order in QCD, our predictions for the total cross sections have significant uncertainties due to the choice of scale in $\alpha_{s}, m_{b}$, and the parton distribution functions. We took the same scale everywhere, viz. the sum of the masses of the produced Higgs bosons. One could therefore infer the existence of squark loop contributions to the total cross section only if it changes the quark loop result by at least a factor of two [34]. However, smaller squark loop contributions might still be visible in some distributions. For example, for large $\tan \beta$ the quark loop contribution is dominated by $b$-quark loops (except for $h h$ production at large $m_{A}$, where $h$ is always SM-like). Since $m_{\widetilde{b}_{1}} \gg m_{b}$, the squark and quark loop functions show quite a different dependence on $\hat{s}$. This is illustrated in Fig. 6, where we show $d \sigma(h h) / d \sqrt{\hat{s}}$ for the point in Fig. 5(a) where $b$ and $\tilde{b}$ loops contribute equally. The solid histogram shows the contribution of $b$ loops only, while the dashed histogram includes squark loops; the total cross section (area under the curves) has been normalized to be the same in both cases. The $b$ loop contribution peaks just beyond the threshold at $\sqrt{\hat{s}}=2 m_{h}$, but the $\widetilde{b}_{1}$ loop contribution clearly shows up as a second peak at $\sqrt{\hat{s}} \simeq 2 m_{\tilde{b}_{1}}$. This distribution is in principle directly measurable if both Higgs bosons decay hadronically (with combined branching ratio $\simeq 80 \%$ ). Given sufficient statistics, one might be able to infer the squark loop contribution in this manner even if the total cross section is dominated by quark loops.

\section{SUMMARY AND CONCLUSIONS}

In this paper we have calculated squark loop contributions to the pair production of two neutral Higgs bosons. If $C P$ is conserved, squark loops contribute only if the two produced Higgs bosons have identical $C P$ quantum numbers. In Sec. II we gave complete analytical expressions that allow the evaluation of these contributions. For completeness we also included expressions for the quark loop contributions [20]. These formulas are written in a completely general fashion with the explicit expressions for the relevant coupling constants in the framework of the MSSM being listed in Appen$\operatorname{dix}$ A.

In Sec. III we showed some numerical results for the MSSM. We found that squark loop contributions can increase the total cross section for the production of two $C P$-even Higgs bosons by more than two orders of magnitude. However, such large contributions are possible only if the following three conditions are satisfied:

The relevant Yukawa coupling must be large. In case of the (s)top, this is always true for at least one of the two $C P$-even Higgs bosons of the MSSM. However, as pointed out quite some time ago [35], in the MSSM (as in other models with more than one Higgs doublet) the bottom Yukawa coupling can also be large, if the vacuum expectation value that gives rise to the mass of the $b$ quark is small. In the MSSM this happens for large values of $\tan \beta$. However, the bottom Yukawa coupling is not expected to exceed that of the top quark.

The lighter of the two superpartner whose Yukawa coupling is large (generally $\widetilde{b}_{1}$ at large $\tan \beta$ and $\tilde{t}_{1}$ at small $\tan \beta$ ) must not be much heavier than the Higgs bosons in the final state. This condition is especially critical at large $\tan \beta$, since here $\tilde{b}_{1}$ loops have to compete with $b$ quark loops. For equal Yukawa couplings (i.e., $\tan \beta \simeq m_{t} / m_{b}$ ), the squared $b$-loop contribution exceeds the squared $t$-loop contribution by a factor of $\sim 50$. If this condition is satisfied, open squark pair production should be detectable at the same collider, unless the squark-LSP mass difference is very small.

The relevant trilinear soft breaking parameters and/or $|\mu|$ must be significantly larger than the mass of the lighter squark eigenstate. This implies that the diagonal entries of the corresponding squark mass matrix must also significantly exceed the smaller eigenvalue of this matrix. This requires a modest amount of fine tuning. However, in the absence of a complete theory of supersymmetry breaking this possibility should not be discounted.

If these three conditions are satisfied, the region $m_{A}$ $\simeq 100 \mathrm{GeV}$ can perhaps even be probed at the next run of the Tevatron collider, using searches for final states with 4 high- $p_{T} \quad b$-jets. Moreover, this process has a big advantage over the $\phi b \bar{b}$ associated production [16], which leads to the same final state: the reconstruction efficiency of Higgs pairs 
is expected to be one order higher than that for $\phi b \bar{b}$ production. This originates from the fact that all $b$-jets coming from Higgs boson pair decays are energetic, while the two associated $b$-jets in the $\phi b \bar{b}$ channel are quite soft. At the same time the process under study has a cross section not much smaller than that for $\phi b \bar{b}$. The reach of the LHC should be much higher, but a quantitative statement will only be possible after a detailed analysis of signal and background [36]. Furthermore, we should point out that our result for the 1-loop cross sections are probably very conservative, since we can expect large QCD corrections, which could increase the cross section by as much as a factor of $\sim 2[7,8,21]$.

Squark loop contributions to the pair production of two $C P$-odd Higgs bosons are more modest in general. In this case trilinear $A \widetilde{q}_{i} \widetilde{q}_{j}^{*}$ couplings contribute only if at least one squark in the loop is a heavy mass eigenstate, which leads to a suppression of the squark contributions. Nevertheless, squark loops can give rise to large enhancements of the $A$ pair production cross section if $m_{\tilde{t}_{1}} \leqslant m_{t}$ and $\tan \beta \sim 5$, which leads to a very small quark loop contribution. However, the total cross section for $A A$ production remains quite small in this case.

In general $A A$ final states, as well as the $h A$ and $H A$ final states, which receive no contributions from squark loops (but do receive Drell-Yan like contributions from light $q \bar{q}$ annihilation [20]), can be significant. Note that in most regions of parameter space the $C P$-odd Higgs boson $A$ is nearly degenerate with one of the two $C P$-even Higgs bosons. This means that often three different channels (e.g., $H H, H A$ and $A A$ ) contribute to essentially the same final state, and therefore have to be added. This obviously increases the chance to detect a signal for Higgs pair production in the $4 b$ channel. At the same time it complicates the interpretation of such a signal, e.g., the extraction of the relevant coupling constants. Nevertheless, we saw in Fig. 6 that the analysis of the $4 b$ invariant mass distribution can help to disentangle the various contributions to the signal. We are therefore hopeful that the search for the pair production of neutral Higgs bosons in the $4 b$ channel will provide information that will help us to pin down the Higgs sector, and perhaps also the squark sector of the theory.

\section{ACKNOWLEDGMENTS}

A.B., M.D., and J.K.M. were supported by FAPESP, Brazil. A.B. would like to thank A. Solomin for the help with the computing facilities. This work was supported by Conselho Nacional de Desenvolvimento Científico e Tecnológico $(\mathrm{CNPq})$, by Fundação de Amparo à Pesquisa do Estado de São Paulo (FAPESP), by Programa de Apoio a Núcleos de Excelência (PRONEX) and by U.S. Department of Energy under the contract DE-AC03-76SF00515.

\section{APPENDIX A: MSSM COUPLING CONSTANTS}

We denote the weak mixing angle and couplings as

$$
s_{W} \equiv \sin \theta_{W}, \quad c_{W} \equiv \cos \theta_{W}, \quad g=e / s_{W}, \quad g_{Z}=g / c_{W} .
$$

We define squark mass eigenstates via

$$
\left(\begin{array}{c}
\widetilde{f}_{1} \\
\widetilde{f}_{2}
\end{array}\right)=\left(\begin{array}{cc}
\cos \theta_{f} & \sin \theta_{f} \\
-\sin \theta_{f} & \cos \theta_{f}
\end{array}\right)\left(\begin{array}{c}
\widetilde{f}_{L} \\
\widetilde{f}_{R}
\end{array}\right), \quad f=u, d,
$$

where the mixing angle $\theta_{f}$ is defined in such way that the mass matrix becomes diagonal with eigenvalues $m_{\tilde{f}_{1}}^{2}$ and $m_{\tilde{f}_{2}}^{2}\left(m_{\tilde{f}_{1}}<m_{\tilde{f}_{2}}\right)$ :

$$
\begin{aligned}
& \left(\begin{array}{cc}
\cos \theta_{f} & \sin \theta_{f} \\
-\sin \theta_{f} & \cos \theta_{f}
\end{array}\right)\left(\begin{array}{cc}
m_{\tilde{f}_{L}}^{2} & m_{\tilde{f}_{L R}}^{2} \\
m_{\tilde{f}_{L R}}^{2 *} & m_{\tilde{f}_{R}}^{2}
\end{array}\right)\left(\begin{array}{cc}
\cos \theta_{f} & -\sin \theta_{f} \\
\sin \theta_{f} & \cos \theta_{f}
\end{array}\right) \\
& =\left(\begin{array}{cc}
m_{\tilde{f}_{1}}^{2} & 0 \\
0 & m_{\tilde{f}_{2}}^{2}
\end{array}\right)
\end{aligned}
$$

with

$$
\begin{gathered}
m_{\tilde{f}_{L}}^{2}=\tilde{m}_{\tilde{f}_{L}}^{2}+m_{f}^{2}+M_{Z}^{2} \cos 2 \beta\left(T_{3}^{f}-Q^{f} s_{W}^{2}\right) ; \\
m_{\tilde{f}_{R}}^{2}=\tilde{m}_{\tilde{f}_{R}}^{2}+m_{f}^{2}+M_{Z}^{2} \cos 2 \beta Q_{f} s_{W}^{2} ; \\
m_{\tilde{f}_{L R}}^{2}= \begin{cases}-m_{u}\left(\mu \cot \beta-A_{u}^{*}\right), & f=u, \\
-m_{f}\left(\mu \tan \beta-A_{f}^{*}\right), & f=d .\end{cases}
\end{gathered}
$$

Squark current eigenstate bilinears can then be expressed in terms of current mass as follows:

$$
\widetilde{f}_{L}^{*} \tilde{f}_{L}=+c_{f}^{2} \widetilde{f}_{1}^{*} \widetilde{f}_{1}-c_{f} s_{f} \widetilde{f}_{1}^{*} \tilde{f}_{2}-c_{f} s_{f} \widetilde{f}_{2}^{*} \widetilde{f}_{1}+s_{f}^{2} \widetilde{f}_{2}^{*} \widetilde{f}_{2}
$$

$$
\tilde{f}_{L}^{*} \widetilde{f}_{R}=+c_{f} s_{f} \widetilde{f}_{1}^{*} \widetilde{f}_{1}+c_{f}^{2} \widetilde{f}_{1}^{*} \widetilde{f}_{2}-s_{f}^{2} \widetilde{f}_{2}^{*} \widetilde{f}_{1}-c_{f} s_{f} \widetilde{f}_{2}^{*} \widetilde{f}_{2}
$$

$$
\begin{aligned}
& \widetilde{f}_{R}^{*} \widetilde{f}_{L}=+c_{f} s_{f} \widetilde{f}_{1}^{*} \widetilde{f}_{1}-s_{f}^{2} \widetilde{f}_{1}^{*} \widetilde{f}_{2}+c_{f}^{2} \widetilde{f}_{2}^{*} \widetilde{f}_{1}-c_{f} s_{f} \widetilde{f}_{2}^{*} \widetilde{f}_{2} ; \\
& \widetilde{f}_{R}^{*} \widetilde{f}_{R}=+s_{f}^{2} \widetilde{f}_{1}^{*} \widetilde{f}_{1}+c_{f} s_{f} \widetilde{f}_{1}^{*} \widetilde{f}_{2}+c_{f} s_{f} \widetilde{f}_{2}^{*} \widetilde{f}_{1}+c_{f}^{2} \widetilde{f}_{2}^{*} \widetilde{f}_{2},
\end{aligned}
$$

with $c_{f}=\cos \theta_{f}$ and $s_{f}=\sin \theta_{f}$ for $f=u, d$.

After these preliminaries we are ready to list the relevant couplings. We list couplings to squark current eigenstates only; these can be converted into couplings to mass eigenstates using Eqs. (A5). 
$H(h, A)$-fermion-fermion

$$
\begin{array}{lll}
\bar{u} u h^{0}: & -\frac{g m_{u} \cos \alpha}{2 M_{W} \sin \beta}, \quad \bar{u} u H^{0}: & -\frac{g m_{u} \sin \alpha}{2 M_{W} \sin \beta}, \\
\bar{u} u A^{0}: & +i \frac{g m_{u}}{2 M_{W}} \cot \beta \gamma_{5}, \quad \bar{d} d h^{0}: & +\frac{g m_{d} \sin \alpha}{2 M_{W} \cos \beta}, \\
\bar{d} d H^{0}: & -\frac{g m_{d} \cos \alpha}{2 M_{W} \cos \beta}, \quad \bar{d} d A^{0}: & +i \frac{g m_{d}}{2 M_{W}} \tan \beta \gamma_{5} .
\end{array}
$$

\section{Gluon-squark-squark}

$$
G^{\mu \alpha} \widetilde{f}_{i}^{*}(p) \widetilde{f}_{i}(k):-i g_{s} \frac{\lambda^{\alpha}}{2}(k-p)^{\mu},
$$

where $k$ and $p$ are the incoming momenta of the squarks $\widetilde{q}_{i}$, with $i=1,2$.

\section{H-squark-squark}

$$
\begin{gathered}
\tilde{u}_{L}^{*} \tilde{u}_{L} H^{0}: \quad-\left(\frac{g m_{u}^{2} \sin \alpha}{M_{W} \sin \beta}+g_{Z} M_{Z} \cos (\alpha+\beta)\left(\frac{1}{2}-\frac{2}{3} s_{W}^{2}\right)\right) \\
\tilde{u}_{R}^{*} \tilde{u}_{R} H^{0}: \quad-\left(\frac{g m_{u}^{2} \sin \alpha}{M_{W} \sin \beta}+\frac{2}{3} g_{Z} M_{Z} \cos (\alpha+\beta) s_{W}^{2}\right) \\
\tilde{u}_{L}^{*} \tilde{u}_{R} H^{0}, \tilde{u}_{R}^{*} \tilde{u}_{L} H^{0}: \quad+\frac{g m_{u}}{2 M_{W} \sin \beta}\left(A_{u} \sin \alpha+\mu \cos \alpha\right) \\
\tilde{d}_{L}^{*} \widetilde{d}_{L} H^{0}: \quad-\left(\frac{g m_{d}^{2} \cos \alpha}{M_{W} \cos \beta}+g_{Z} M_{Z} \cos (\alpha+\beta)\left(-\frac{1}{2}+\frac{1}{3} s_{W}^{2}\right)\right) \\
\tilde{d}_{R}^{*} \widetilde{d}_{R} H^{0}: \quad-\left(\frac{g m_{d}^{2} \cos \alpha}{M_{W} \cos \beta}-\frac{1}{3} g_{Z} M_{Z} \cos (\alpha+\beta) s_{W}^{2}\right)
\end{gathered}
$$

$$
\widetilde{d}_{L}^{*} \widetilde{d}_{R} H^{0}, \widetilde{d}_{R}^{*} \widetilde{d}_{L} H^{0}: \quad+\frac{g m_{d}}{2 M_{W} \cos \beta}\left(A_{d} \cos \alpha+\mu \sin \alpha\right)
$$

\section{h-squark-squark}

$$
h \widetilde{f}^{*} \widetilde{f}=H \widetilde{f}^{*} \widetilde{f}\{\sin \alpha \rightarrow \cos \alpha, \cos \alpha \rightarrow-\sin \alpha, \sin (\alpha+\beta)
$$$$
\rightarrow \cos (\alpha+\beta), \quad \cos (\alpha+\beta) \rightarrow-\sin (\alpha+\beta)\} .
$$

\section{A-squark-squark}

$$
\begin{array}{ll}
\tilde{u}_{L}^{*} \tilde{u}_{R} A^{0}: & -i \frac{g m_{u}}{2 M_{W}}\left(A_{u} \cot \beta-\mu\right), \\
\tilde{u}_{R}^{*} \tilde{u}_{L} A^{0}: & +i \frac{g m_{u}}{2 M_{W}}\left(A_{u} \cot \beta-\mu\right), \\
\widetilde{d}_{L}^{*} \widetilde{d}_{R} A^{0}: & -i \frac{g m_{d}}{2 M_{W}}\left(A_{d} \tan \beta-\mu\right),
\end{array}
$$

$$
\widetilde{d}_{R}^{*} \widetilde{d}_{L} A^{0}: \quad+i \frac{g m_{d}}{2 M_{W}}\left(A_{d} \tan \beta-\mu\right) .
$$

Gluon-gluon-squark-squark

$$
g_{\mu}^{\alpha} g^{\mu \beta} \tilde{q}_{i}^{*} \tilde{q}_{i}: g_{s}^{2} \frac{\lambda^{\alpha}}{2} \frac{\lambda^{\beta}}{2}
$$

\section{H-H-squark-squark}

$$
\begin{gathered}
\tilde{u}_{L}^{*} \tilde{u}_{L}\left(H^{0}\right)^{2}: \quad-\left(\frac{g^{2} m_{u}^{2} \sin ^{2} \alpha}{4 M_{W}^{2} \sin ^{2} \beta}+\frac{1}{4} g_{Z}^{2}\left(T_{3 u}-s_{W}^{2} Q_{u}\right) \cos 2 \alpha\right), \\
\tilde{u}_{R}^{*} \tilde{u}_{R}\left(H^{0}\right)^{2}: \quad-\left(\frac{g^{2} m_{u}^{2} \sin ^{2} \alpha}{4 M_{W}^{2} \sin ^{2} \beta}+\frac{1}{4} g_{Z}^{2} s_{W}^{2} Q_{u} \cos 2 \alpha\right), \\
\tilde{d}_{L}^{*} \tilde{d}_{L}\left(H^{0}\right)^{2}: \quad-\left(\frac{g^{2} m_{d}^{2} \cos ^{2} \alpha}{4 M_{W}^{2} \cos ^{2} \beta}+\frac{1}{4} g_{Z}^{2}\left(T_{3 d}-s_{W}^{2} Q_{d}\right) \cos 2 \alpha\right), \\
\tilde{d}_{R}^{*} \tilde{d}_{R}\left(H^{0}\right)^{2}: \quad-\left(\frac{g^{2} m_{d}^{2} \cos ^{2} \alpha}{4 M_{W}^{2} \cos ^{2} \beta}+\frac{1}{4} g_{Z}^{2} s_{W}^{2} Q_{d} \cos 2 \alpha\right) . \\
h h \tilde{q} \tilde{q}=H H \tilde{q} \tilde{q}\{\sin \alpha \rightarrow-\cos \alpha, \cos \alpha \rightarrow \sin \alpha, \cos 2 \alpha \\
\rightarrow-\cos 2 \alpha\} .
\end{gathered}
$$

\section{H-h-squark-squark}

$H h \tilde{q} \tilde{q}=H H \tilde{q} \widetilde{q}\left\{\cos 2 \alpha \rightarrow-\sin 2 \alpha, \sin ^{2} \alpha \rightarrow \sin 2 \alpha, \cos ^{2} \alpha\right.$ $\rightarrow-\sin 2 \alpha\}$.

\section{A-A-squark-squark}

$$
A A \tilde{q} \tilde{q}=H H \tilde{q} \tilde{q}\{\sin \alpha \rightarrow-\cos \beta, \cos \alpha \rightarrow \sin \beta, \cos 2 \alpha
$$$$
\rightarrow-\cos 2 \alpha\} \text {. }
$$

\section{Higgs $^{0}$-boson-Higgs ${ }^{0}$-boson- Higgs $^{0}$-boson}

$$
\left(H^{0}\right)^{3}: \quad-\frac{g_{Z}}{4} M_{Z} \cos 2 \alpha \cos (\alpha+\beta)
$$

$\left(h^{0}\right)^{3}: \quad-\frac{g_{Z}}{4} M_{Z} \cos 2 \alpha \sin (\alpha+\beta)$,

$\left(A^{0}\right)^{2} H^{0}: \quad+\frac{g_{Z}}{4} M_{Z} \cos 2 \beta \cos (\alpha+\beta)$,

$\left(A^{0}\right)^{2} h^{0}: \quad-\frac{g_{Z}}{4} M_{Z} \cos 2 \beta \sin (\alpha+\beta)$, 


$$
\begin{aligned}
\left(h^{0}\right)^{2} H^{0}: & -\frac{g_{Z}}{4} M_{Z}[2 \sin 2 \alpha \sin (\alpha+\beta) \\
& -\cos 2 \alpha \cos (\alpha+\beta)], \\
\left(H^{0}\right)^{2} h^{0}: \quad & +\frac{g_{Z}}{4} M_{Z}[2 \sin 2 \alpha \cos (\alpha+\beta) \\
& +\cos 2 \alpha \sin (\alpha+\beta)] .
\end{aligned}
$$

\section{Z-Higgs-boson-Higgs-boson}

$$
\begin{array}{ll}
h^{0}(p) A^{0}(k) Z_{\mu}: & +\frac{i g_{z}}{2} \cos (\alpha-\beta)(k-p)^{\mu}, \\
h^{0}(p) A^{0}(k) Z_{\mu}: & +\frac{i g_{z}}{2} \sin (\alpha-\beta)(k-p)^{\mu} .
\end{array}
$$

\section{APPENDIX B: CONVENTIONS}

We chose the following polarization vectors in our calculations

$$
\epsilon_{1}^{+}=\epsilon_{2}^{-}=\frac{1}{\sqrt{2}}(0,-1,-i, 0),
$$

[1] E. Witten, Nucl. Phys. B188, 513 (1981).

[2] For a review, see H. E. Haber and G. L. Kane, Phys. Rep. 117, 75 (1985).

[3] See, e.g., J. F. Gunion, H. E. Haber, G. L. Kane, and S. Dawson, The Higgs Hunter's Guide (Addison-Wesley, Reading, MA, 1990).

[4] V. Barger, K. Cheung, R. J. N. Phillips, and A. Stange, Phys. Rev. D 47, 3041 (1993); A. Djouadi, J. Kalinowski, and P. Zerwas, Z. Phys. C 57, 569 (1993); A. Yamada, ibid. 61, 247 (1994).

[5] H. Georgi, S. L. Glashow, M. E. Machacek, and D. V. Nanopoulos, Phys. Rev. Lett. 40, 692 (1978); S. F. Novaes, Phys. Rev. D 27, 2211 (1983).

[6] J. F. Gunion and H. E. Haber, Nucl. Phys. B278, 449 (1986).

[7] A. Djouadi, M. Spira, and P. M. Zerwas, Phys. Lett. B 264, 440 (1991); S. Dawson, Nucl. Phys. B359, 283 (1991); M. Spira, A. Djouadi, D. Graudenz, and P. M. Zerwas, ibid. B453, 17 (1995).

[8] S. Dawson, A. Djouadi, and M. Spira, Phys. Rev. Lett. 77, 16 (1996).

[9] J. F. Gunion, G. Gamberini, and S. F. Novaes, Phys. Rev. D 38, 3481 (1988).

[10] A. Djouadi, Phys. Lett. B 435, 101 (1998).

[11] For a review, see J. F. Gunion, A. Stange, and S. Willenbrock, in Electroweak Symmetry Breaking and New Physics at the TeV Scale, edited by T. L. Barklow, S. Dawson, H. E. Haber, and J. L. Siegrist (World Scientific, Singapore, 1996), hep-ph/9602238.

[12] A. Stange, W. Marciano, and S. Willenbrock, Phys. Rev. D 49,

$$
\epsilon_{1}^{-}=\epsilon_{2}^{+}=\frac{1}{\sqrt{2}}(0,1,-i, 0),
$$

where the first gluon is moving along the $z$ axis.

We have used scalar $C_{0}$ and $D_{0}$ Passarino-Veltman functions in our analytical formulas which are expressed as:

$$
\begin{aligned}
& C_{0}[p 10, p 12, p 20, m 0, m 1, m 2] \\
& =\left(i \pi^{2}\right)^{-1} \int d^{4} q\left(\left[q^{2}-m_{0}^{2}\right]\left[\left(q+p_{1}\right)^{2}-m_{1}^{2}\right]\right. \\
& \left.\times\left[\left(q+p_{2}\right)^{2}-m_{2}\right]\right)^{-1} \\
& D_{0}[p 10, p 12, p 23, p 30, p 20, p 13, m 0, m 1, m 2, m 3] \\
& =\left(i \pi^{2}\right)^{-1} \int d^{4} q\left(\left[q^{2}-m_{0}^{2}\right]\left[\left(q+p_{1}\right)^{2}-m_{1}^{2}\right]\right. \\
& \left.\quad \times\left[\left(q+p_{2}\right)^{2}-m_{2}\right]\left[\left(q+p_{3}\right)^{2}-m_{3}^{2}\right]\right)^{-1} .
\end{aligned}
$$

Our convention for the scalar arguments is $p i 0=p_{i}^{2}, p i j$ $=\left(p_{i}-p_{j}\right)^{2}$, and $m i=m_{i}$.
1354 (1994); A. Belyaev, E. Boos, and L. Dudko, Mod. Phys. Lett. A 10, 25 (1995).

[13] CMS Collaboration, S. Abdullin et al., CMS-NOTE-1998-006, hep-ph/9806366.

[14] J. Dai, J. F. Gunion, and R. Vega, Phys. Lett. B 315, 355 (1993); 345, 29 (1995)

[15] A. Djouadi, J. L. Kneur, and G. Moultaka, Phys. Rev. Lett. 80, 1830 (1998); A. Dedes and S. Moretti, Phys. Rev. D 60, 015007 (1999); G. Bélanger, F. Boudjema, and K. Sridhar, hep-ph/9904348.

[16] J. Lorenzo Diaz-Cruz, Hong-Jian He, Tim Tait, and C. P. Yuan, Phys. Rev. Lett. 80, 4641 (1998); C. Balazs, J. L. DiazCruz, H. J. He, T. Tait, and C. P. Yuan, Phys. Rev. D 59, 055016 (1999).

[17] M. Drees, M. Guchait, and P. Roy, Phys. Rev. Lett. 80, 2047 (1998); 81, 2394(E) (1998).

[18] A. Djouadi, W. Kilian, M. Muhlleitner, and P. M. Zerwas, DESY 99/033, hep-ph/9904287.

[19] O. J. P. Éboli, G. C. Marques, S. F. Novaes, and A. A. Natale, Phys. Lett. B 197, 269 (1987); D. D. Dicus, C. Kao, and S. Willenbrock, ibid. 203, 457 (1988); E. W. N. Glover and J. J. van der Bij, Nucl. Phys. B309, 282 (1988).

[20] T. Plehn, M. Spira, and P. M. Zerwas, Nucl. Phys. B479, 46 (1996); B531, 655(E) (1998).

[21] S. Dawson, S. Dittmaier, and M. Spira, Phys. Rev. D 58, 115012 (1998).

[22] R. Mertig, M. Bohm, and A. Denner, Comput. Phys. Commun. 64, 345 (1991)

[23] G. Passarino and M. Veltman, Nucl. Phys. B160, 151 (1979). 
[24] CTEQ Collaboration, H. Lai, J. Huston, S. Kuhlmann, F. Olness, J. Owens, D. Soper, W.-K. Tung, and H. Weerts, Phys. Rev. D 55, 1280 (1997).

[25] Of course, squark masses below $50 \mathrm{GeV}$ are excluded experimentally. We extend the curves in Fig. 3 to such small values of $m_{\tilde{q}}$ since the shapes of these curves remain the same when $m_{\tilde{q}}, \quad m_{h}$ and $\sqrt{\hat{s}}$ are all scaled up by the same factor. In some regions of parameter space $H \rightarrow \tilde{t}_{1} \tilde{t}_{1}^{*}$ or $H \rightarrow \widetilde{b}_{1} \widetilde{b}_{1}^{*}$ decays are still possible; see A. Bartl, H. Eberl, K. Hidaka, T. Kon, W. Majerotto, and Y. Yamada, Phys. Lett. B 402, 303 (1997).

[26] ALEPH Collaboration, R. Barate et al., Phys. Lett. B 440, 419 (1998); L3 Collaboration, M. Acciarri et al., ibid. 436, 389 (1998); OPAL Collaboration, G. Abbiendi et al., Eur. Phys. J. C 7, 407 (1999).

[27] Y. Okada, M. Yamaguchi, and T. Yanagida, Prog. Theor. Phys. 85, 1 (1991); H. E. Haber and R. Hempfling, Phys. Rev. Lett. 66, 1815 (1991).

[28] J. Ellis, G. Ridolfi, and F. Zwirner, Phys. Lett. B 257, 83 (1991); 262, 477 (1991).

[29] M. Drees and M. M. Nojiri, Phys. Rev. D 45, 2482 (1992).

[30] The expressions in M. Carena, J. Espinosa, M. Quirós, and C. Wagner, Phys. Lett. B 355, 209 (1995); M. Carena, M. Quirós, and C. Wagner, Nucl. Phys. B461, 407 (1996) that include RG improvement and leading 2-loop corrections are not applicable for large splitting between the squark masses; such cases are important for us. Note also that much of these effects can be incorporated into the 1-loop expression [see [29] and H. E.
Haber, R. Hempfling, and A. Hoang, Z. Phys. C 75, 539 (1997)] by using running quark masses at the appropriate scale.

[31] OPAL Collaboration, G. Abbiendi et al., Phys. Lett. B 456, 95 (1999).

[32] Searches at the Tevatron [D0 Collaboration, S. Abachi et al., Phys. Rev. Lett. 76, 2222 (1996); C. Holck for the CDF Collaboration, in the Proceedings of the 1999 DPF conference, Los Angeles, CA, 1999, hep-ex/9903060] for a single squark mass eigenstate decaying into a stable neutralino plus a light quark exclude squark masses up to $\sim 120 \mathrm{GeV}$, but only if the neutralino mass is less than $\sim 50 \mathrm{GeV}$. Under the same assumptions the LEP search limit remains valid as long as the squark-neutralino mass splitting exceeds $\sim 5 \mathrm{GeV}$.

[33] Preliminary results from the LEP run at $\sqrt{s}=189 \mathrm{GeV}$ [M. Pepe-Altarelli for the ALEPH Collaboration, hep-ex/9904006] indicate $m_{h} \geqslant 95 \mathrm{GeV}$ for SM-like $h$. This would exclude only the very top of the curves in Fig. 4(b), reducing the maximal squark loop contribution in this case by about a factor of 2 .

[34] We are assuming here that the relevant Yukawa couplings, i.e., the Higgs boson mixing angles $\alpha$ and $\beta$, are known from other measurements; otherwise the uncertainty in the quark loop contribution is much larger.

[35] R. M. Barnett, G. Senjanovic, and D. Wyler, Phys. Rev. D 30, 1529 (1984); P. N. Pandita, Phys. Lett. 151B, 51 (1985).

[36] A. Belyaev et al. (in preparation). 